\title{
DIVERSE TECHNIQUES FOR GEOMETRIC FEATURES EXTRACTION AND IDENTIFICATION OF GESTURE RECOGNITION
}

\author{
Noor A. Ibraheem \\ Computer Science Dept, College of Science for Women, University of Baghdad, Iraq
}

\begin{abstract}
Gestures recognition system registered a prominent stage for multiple utilization in our numerous life functionality in a manner that leads to new human machine interfaces (HMI) to connect with electronic apparatus technologies.Gesture recognition system is the most effective field that witnessed tremendous growth and their applications are spread to comprise smartphones, 3D vision, gaming, healthcare, etc. Gesture recognition system consists of four main steps which are hand shape modeling, extracting hand geometric parameters such as palm center, wrist, determining fingers' base and fingertip, features vector extraction, and finally classification.In this paper we will demonstrate some new techniques that deal with the extracting of geometric parameters and features vector extraction stages.
\end{abstract}

\section{KEYWORDS}

Hand parameters, Geometric features, Features vector detection, Finger identification.

\section{INTRODUCTION}

Who can imagine or even predict that the gestures recognition system can reach to this prominent status of super utilization in all our life complicated functionalitywhich leads to new human machine interfaces (HMI) to connect with electronic apparatus nowadays. According to the worldwide market research company, Global Industry Analysts (GIA), thegesture recognition will invade the world by 2020 . With the passage of time, demanded is filled by the need for natural, creative, intuitive, and real interfaces in portable and handheld devices such as smartphones, 3D vision, gaming, healthcare, sensor technology for wearable devices, automation , etc., moreover, this will develop new innovative in various technology areas including robots and machine control [1].Figure 1 demonstrates the applicable of gesture control in the automotive infotainment market for the latest iDrive launch from BMW.

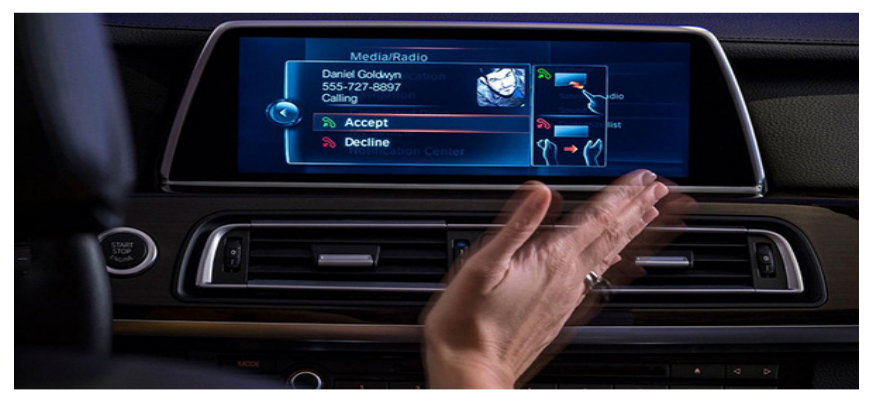

Figure 1: Touch-less gesture control applied in iDrive launch from BMW[1]. 


\section{Computer Applications: An International Journal (CAIJ), Vol.3, No.3, August 2016}

Wearable devices that demanded sensors and circuitry components required small, and a tiny usage of power to maintain battery life, however, these equipment neededamelioration in speed, accuracy, and easy in design which considered as the main challenges of any gesture system. These devices have proved its efficiency as a solution in many medical applications [1].Figure 2 shows game controlling using hand gestures [1].

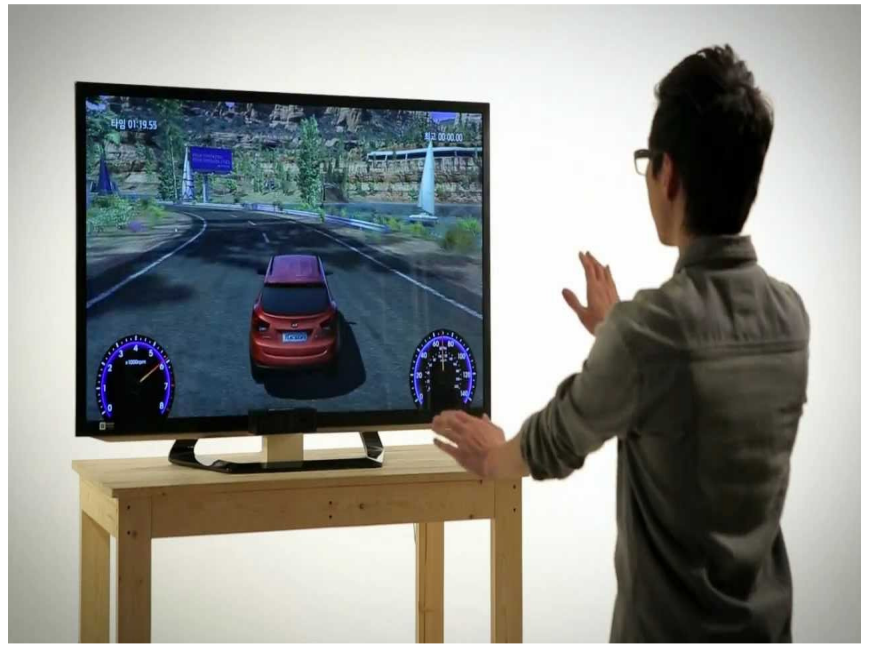

Figure 2: game controlling using hand gestures [1].

However, Gesture challenges come from the facts that gesture is an articulated object, besides environment's variations, and hand occlusion [3][9]. The solution to these problems required suitable and robust features extraction. However, features extraction phase has some steps that should be applied properly to guarantee perfect features vector, these steps comprises some hand parameters such as the locating of palm center, wrist area, fingertip and finger base parameters after modeling the hand properly [2][3][4][5]. Certainly, the use of glove based techniques will simplify the process of extracting the features by offering an exact position and orientation of the hand which in turn will facilitate hand tracking [6], in this paper; we considered the vision based techniques which required a natural interaction with the computer without cost to be mentioned [7]. After extracting the features, a suitable recognition modeling technique is selected to identify the fingers and recognize the gesture such as Neural Network (NN) [8], genetic Algorithms [11],Hidden Markov Models (HMMs) [10],Gaussian model [2][4][12]. Recently a lot of survey that demonstrates the details of gesture recognition systems and their applications are available in the following references [13][14][15][16]. This paper is organized as follows; Section 2 explained Detecting Hand Parameters, Section 3 demonstrated the Representing Finger Features, Features Vector Extraction is provided in Section 4, Summary and conclusions are presented in Section 5.

\section{Detecting Hand Geometric Parameters}

In order to extract the main features of the input hand gesture, the main effective parameters should be identified correctly to simplify features vector process, these geometric parameters include wrist area, palm center, fingers tip and finger base which considered as the reference points necessary to accomplish the extraction process properly.

In [2] fingers and wrist reference points are extracted by locating fingers' and wrist reference points depends on the maximum half diameter of the circle $\mathrm{R}$ value attained during the extraction of final palm area using Fingers and Wrist Reference points Extraction (F_WRPE) algorithm. The 
algorithm traces each pixel on palm center area circle in a clockwise direction from 0 o to $360 \mathrm{o}$ degree [2].

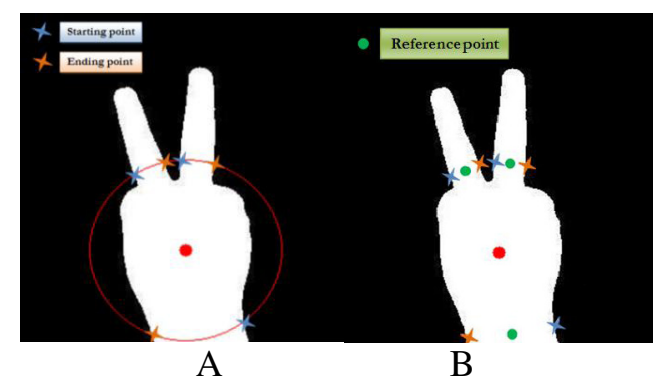

\section{A.Identifying the border points for the wrist and fingers areas. B. Computing the reference points corresponding to the border points.}

Figure 3: assigning reference points for the wrist and fingers areas [2].

Other method to detect hand parameters applied the following calculation to extract the palm area as explained in the following formula [4]:

$$
P_{\text {center }}=\sum_{j \in t_{p}} P(j) / t_{p}
$$

Where $\mathrm{P}$ represents the palm area and tp represents the total number of pixels in palm p.while the wrist area is located using the circular projection method which can detect the area regardless the orientation of the hand. the following figure depicts the extraction of these parameters.



A: locating the starting and ending boundaies of the wrist

$B$ : wrist boundaries and centre after localizing

Figure 4: locating the wrist area as well as the wrist centeralong with a palm area [4].

Researchers in [5] calculated the center of the segmented hand region and applied the center-ofgravity $(\mathrm{CoG})$ method to compute the center position of highest magnitude's pixel, then the finger locationcan be detected from the contour by computing the convex hull of the segmented hand.

\section{REPRESENTING Finger FEATURES}

For any extraction of the fingers parameters, two main steps are required; the extraction of the finger base, and the FingerTip. Finger Base Detection (FBD) and FingerTip Detection (FTD) algorithm have been applied in [2] where the slope of the line between the palm center and the StartPoint, and palm center with the EndPoint are computed to calculate the slope difference 
between border points and generate the BaseDistance, this process continues by decreasing $\mathrm{R}$ and tracing the left side and right side of the reference points, this loop stops when an approximated distance of finger base is generated. For extracting the fingertip, the same algorithm is applied in ascendingorder by increasing $\mathrm{R}$ value at each iteration,figure 5 depicts the steps of identifying fingers base and fingertip respectively.

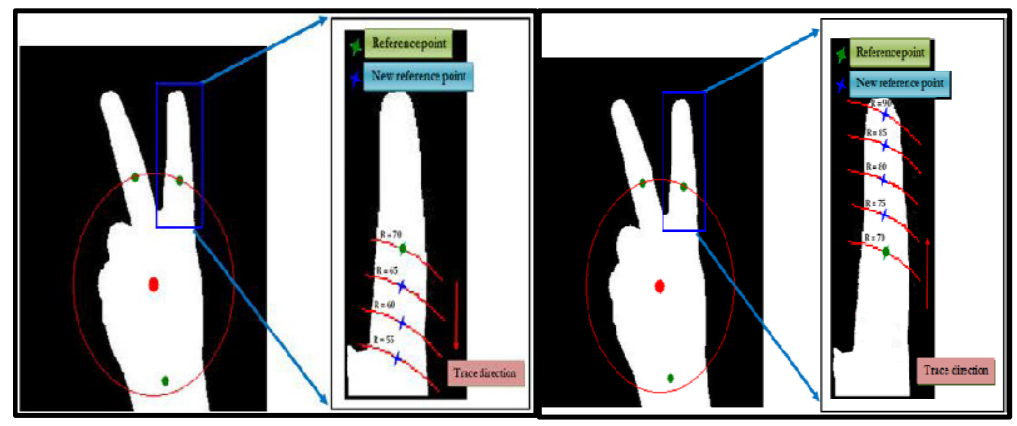

$A, B$. Hand fingers after locating wrist and fingers areas.A. Section part of the right finger to explain finger base process. B. Section part of the right finger to explain fingertip process.

Figure5: Overview of and fingertip detection algorithm [2].

While in [4] The fingertips, finger centers and finger bases as well as parametersand palm related parameterscan be detected by doing some calculation from the resulting data; these data can come from (1), (2), and (3) as follows:

$$
\begin{aligned}
& F_{i}(\text { tip })=\max _{j \in t_{g}}\left|P_{\text {centre }}-F_{i}(j)\right|, \forall i \in g \\
& F_{i}(\text { center })=\sum_{j \in t_{g}} F_{i}(j) / t_{g}, \forall i \in g \\
& F_{i}(\text { base })=F_{i}(\text { center })+\left(F_{l}(\text { tip }) \widehat{\left.-F_{l}(\text { center })\right) * w, ~}\right. \\
& \forall i \in g
\end{aligned}
$$

Where $\mathrm{P}$ and $\mathrm{F}$ represent the palm and finger respectively, $\mathrm{g}$ is number of divided groups, Fi(j) represents the pixel $j$ of the finger group $i$, tg represents the number of total pixels ingroup $g$, and $(\widehat{\mathrm{B}})$ is the reflection operation.

While the suggested method in [5] defined the fingertip as the center positions between the start point and the end point of the selected defect area, and an accurate fingertip points are calculated by constructing an ellipse that fitted to the contour around filtered finger point using an enhanced least-squares fitting of ellipse (EDFE) method, figure 6 demonstrates the extraction of hand fingertips. 


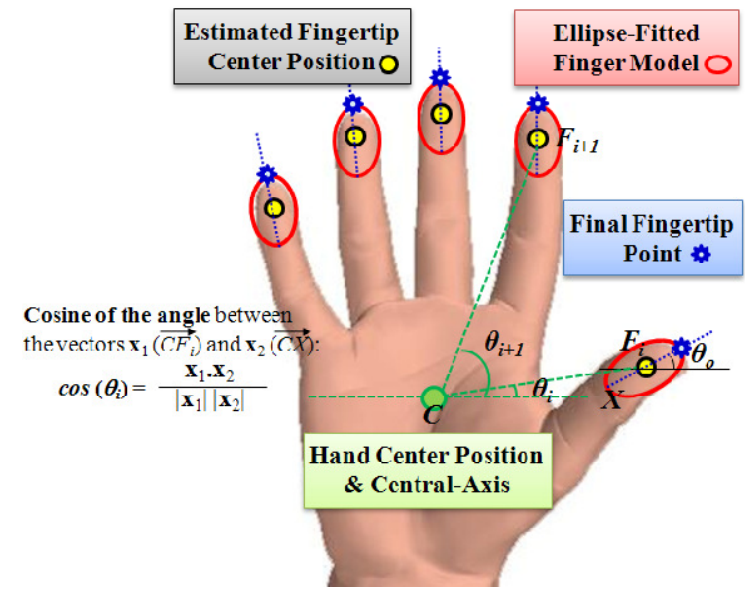

Figure6: The structure of fingertip extraction [5].

\section{FeATURES Vector EXTRACTION}

The most crucial step in any gesture recognition system is the perfect calculating of the features vector in a manner that comprises all gesture's database and combines all the feature space [3]. It is important to indicate the smaller the size of features vector the better for the recognition process. In [2] Two features have been performed; 1) Finger Base Angle (FBA), and 2) Finger Projected Distance (FPD) feature, where the former feature calculates the angle between the two vectors formed between palm center and finger base, while thelatter feature represents the distance of the vertical projection of the finger base and the palm center.

Researchers in [4] suggested four features to recognize fingers and hand gestures accurately, these features are; 1) PD distance between finger base and hand line, 2) BA angle between finger base and hand line, 3) BsA angle between fingers bases and palm center, 4) line between the nearest border pixel to the base of the finger and the thresholding of border pixel.

For a natural and meaningful representation of dynamic gesture recognition, [5] applied geometrical features of hand and vector angle value $(\theta \mathrm{i})$ to extract the thumb, fingertip and finger base. The thumb is identified within the angle range in a counterclockwise direction from the central axis of the hand gesture; $(\theta 0)$ which is the fingertip orientation represents the angle between horizontal east-west line and the largest axis of the suited constructed ellipse [5].

\section{CONCLuSion}

The recent trends of the world are moving towards the natural interaction and free-cumbering interaction with these human-manufacture devices. Gesture recognition system is the most effective field that witnessed such developments. Gesture recognition system consists of some steps, in this paper we explained and discussed some new techniques that deals with the one of these steps which is extracting hand parameters such as palm center, wrist area, fingertip and finger base reference points, which are essential for features vector extraction. As well as we demonstrated the vision based techniques which required a statistical calculation but on the other handthey provide a free communicating with sensors or some other hardware devices. 


\section{ACKNOWLEDGMENT}

We would like to present our thanks to the experts who have contributed towards developing this work.

\section{REFERENCES}

[1] Website:http://www.digikey.com/en/articles/techzone/2016/may/smart-sensors-light-the-waytowards-gesture-recognition

[2] RafiqulZaman Khan, Noor Adnan Ibraheem,"Genetic Shape Fitting for Hand Gesture Modeling and Feature Extraction using Variable Length Chromosome", British Journal of Science, (ISSN 20473745), Volume 10 (1), UK, December 2013

[3] Noor A. Ibraheem, "Finger Identification and Gesture Recognition Using Gaussian Classifier Model", International Journal of Applied Engineering Research, Volume 11(10), pp. 6924-6931, 2016.

[4] Mokhtar M. Hasan, and Pramod K. Mishra, "Novel Algorithm for Multi Hand Detection and Geometric Features Extraction and Recognition”, International Journal of Scientific and Engineering Research, Vol. 3 (5), pp. 1- 11, 2012.

[5] Lae-Kyoung Lee, Su-Yong An, and Se-Young Oh, "Robust FingertipExtraction with Improved Skin Color Segmentation for Finger Gesture Recognition in Human-Robot Interaction", WCCI 2012, IEEE World Congress on Computational Intelligence, June, 2012 Australia.

[6] Mr. Sudarshan G. Ghuge, Mr.Santosh G. Karkhile, "Operating an Application Using Hand Gesture Recognition System”, International Journal of Innovative Research in Computer and Communication Engineering, Vol. 4(2), February 2016.

[7] Zhou Ren ,JingjingMeng ,Junsong Yuan, Zhengyou Zhang ,"Robust Hand Gesture Recognition with Kinect Sensor”, ACM, November 28-December 1, 2011, USA.

[8] HaithamBadi, Sameem Abdul Kareem and Sabah Husien,"Feature Extraction Technique for Static Hand GestureRecognition”, GCSR Recent Trends in Hand Gesture Recognition, vol3.ch2, pp. 19-41, 2015, Doi: $10.15579 / \mathrm{gcsr}$.

[9] Mokhtar M. Hasan and Pramod K. Mishra, "Robust Gesture Recognition Using Gaussian Distribution for Features Fitting", International Journal of Machine Learning and Computing, Vol. 2, No. 3, June 2012.

[10] Lawrence R. Rabiner, 1989. A Tutorial on Hidden Markov Models and Selected Applications in Speech Recognition, Proceedings of the IEEE, vol. 77 (2), pp. 257 - 286.

[11] Noor A. Ibraheem, Mokhtar M. hasan, Shaima M. "Automatic Block Selection for Synthesizing Texture Images using Genetic Algorithms”, Baghdad Science Journal, University of Baghdad, Iraq, vol. 6 (4): 822-830, Dec. 2009.

[12] Mokhar M. Hasan\#1, Pramod K. Mishra, "Features Fitting using Multivariate Gaussian Distribution for Hand Gesture Recognition”, International Journal of Computer Science \& Emerging Technologies, Volume 3(2), April 2012.

[13] Noor A. Ibraheem, Rafiqul Z. Khan,"Vision Based Gesture Recognition Using Neural Networks Approaches: A Review", International Journal of human Computer Interaction (IJHCI), Volume 3(1), 2012

[14] RafigulZaman Khan, Noor Adnan Ibraheem, "Survey on Gesture Recognition for Hand Image Postures", Journal of Computer and Information Science(ISSN: 1913-8989),Volume 5 (3), pp. 110121, Canada, May 2012. Doi: 10.5539/cis.v5n3p110.

[15] RafigulZaman Khan, Noor Adnan Ibraheem, "Hand Gesture Recognition: A Literature Review", International Journal of Artificial Intelligence \& Applications, Volume 3 (4), pp. 161-174, India, July 2012. Doi: 10.5121/ijaia.2012.3412.

[16] Mokhtar M. Hasan\&Pramod K. Mishra, "Hand Gesture Modeling and Recognition using Geometric Features: A Review", Canadian Journal on Image Processing and Computer Vision Vol. 3, No. 1. 2012. 